\title{
Padronização da densidade mineral óssea (DMO) do acessório do carpo em eqüinos atletas da raça Quarto de Milha
}

\author{
Padronization of bone mineral density (BMD) of the acessory carpus in Quarter Horse equines
}

\author{
Luiz Carlos Vulcano ${ }^{1}$ Rodrigo Guerrero Mendes ${ }^{1}$ Carmen Lice Buchmann de Godoy ${ }^{2}$ \\ Vania Maria Vasconcelos Machado ${ }^{1}$ Alexandre Luiz da Costa Bicudo ${ }^{1}$
}

\section{RESUMO}

Eqüinos da raça Quarto de Milha, 30 machos e 30 fêmeas com idade entre 42 e 48 meses, apresentando a epífise distal do rádio fechada, em plena atividade esportiva, foram analisados quanto à densidade mineral óssea (DMO) do acessório do carpo, tendo os valores expressos em milímetros de alumínio ( $m m A l)$. As radiografias da estrutura óssea, juntamente com uma escala de alumínio (penetrômetro) foram analisadas por meio de um programa computacional, especialmente desenvolvido para medida da densidade óptica em imagem radiográfica. $O$ valor médio encontrado para as

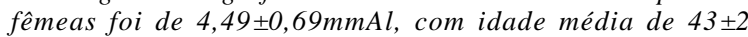
meses e, para os machos, de 4,43 $\pm 0,81 \mathrm{mmAl}$, com idade média de $45 \pm 2$ meses, não havendo diferença significativa na DMO entre os sexos.

Palavras-chave: radiologia, eqüinos, densitometria óssea.

\section{ABSTRACT}

The bone mineral density (BMD) of the accessory carpal bone was measured (in aluminum millimeters - $\mathrm{mmAl}$ ) in 30 female and 30 male Quarter Horse equines aging between 42 and 48 months that presented distal epiphysis of the radio closed, in full activity, using optical densitometry in radiographic images. Evaluation was made using a software specially developed for optical density measurement in $X$-ray films, containing the radiographic image of the accessory carpus bone, the region of soft parts adjacent to the accessory carpus and the degrees of an aluminum step wedge, used to measure $B M D$ value (average of the interest region established on the accessory carpus bone corresponding to the value on the step wedge in millimeters/aluminum ( $\mathrm{mmAl})$ ). BMD values found for female were $4.49 \pm 0.69 \mathrm{~mm}$ Al with age of $43 \pm 2$ months $e$ for male $4.43 \pm 0.8 \mathrm{~mm}$ Al, with average age $45 \pm 2$ months not show differences statistically significant in BMD values among sexes.

Key words: radiology, equine, bone densitometry.

\section{INTRODUÇÃO}

A primeira raça de eqüinos que se desenvolveu na América foi a Quarto de Milha (QM), iniciando-se nos Estados Unidos por volta do ano de 1600. O plantel brasileiro hoje é composto, segundo dados fornecidos pelo Stud Book da ABQM (Associação Brasileira de Criadores de Cavalos Quarto de Milha), atualizados até o início do ano de 2003, por 284.800 animais registrados.

A raça se caracteriza por extrema docilidade, partida rápida, parada brusca, grande capacidade em alternar a direção e enorme habilidade de girar sobre si mesmo. Considerada a mais versátil do mundo, é utilizada em várias modalidades de trabalho e corrida, sendo o cavalo oficial do rodeio completo segundo a ABQM. Assim sendo, estas qualidades da raça justificam estudos visando à avaliação da estrutura óssea destes animais, como a densitometria mineral óssea.

Estudos em eqüinos sugeriram que o exercício levava a valores diferentes da densidade mineral óssea (JEFFCOTT, 1990). Na medicina humana, estudos demonstraram uma correlação direta entre a incidência de fraturas e o baixo conteúdo mineral do esqueleto (O’CALLAGHAN 1991).

Para uma avaliação da densidade mineral óssea (DMO) dos animais em desenvolvimento, tornase necessário o emprego de técnica de medidas in vivo (MEAKIM et al., 1981). Dentre as diversas técnicas

${ }^{1}$ Departamento de Radiologia e Reprodução Animal, Faculdade de Medicina Veterinária e Zootecnia (FMVZ), Universidade Estadual Paulista (UNESP). Rua Tiradentes, 67, 18611-840, Botucatu, São Paulo, Brasil. E-mail vulcano@fmvz.unesp.br. Autor para correspondência.

${ }^{2}$ Departamento de Clínica de Grandes Animais, Universidade Federal de Santa Maria (UFSM), Santa Maria, RS, Brasil. 
desenvolvidas, encontra-se a densitometria óptica em imagem radiográfica, a qual pode ser utilizada para uma análise seqüencial da massa óssea em animais em treinamento a campo, com um custo menor que as demais metodologias, tendo, como fator preponderante, a precisão (VULCANO et al., 2000). Esta metodologia, de baixo custo, requer padronizações acuradas, tais como o uso de padrão de referência, técnica radiológica apropriada e posicionamento específico do material a ser analisado (ANDERSON et al., 1966 e SCHNEIDER, 1984).

Utilizando-se desta técnica, MEAKIM et al. (1981) avaliaram o conteúdo mineral ósseo do III metacarpiano de cadáveres de eqüinos. Dos membros radiografados, amostras ósseas de $2 \mathrm{~cm}$ comprimento em cortes transversais foram retiradas no seu terço médio, secas, pesadas, incineradas e novamente pesadas. Os valores foram expressos em gramas de cinzas ósseas por $2 \mathrm{~cm}$ da secção óssea do III metacarpiano definido como conteúdo mineral ósseo. Estes valores foram confrontados com seus valores de espessura em milímetros de alumínio, obtidos através das curvas de transmitância de luz das imagens radiográficas dos degraus de um penetrômetro de alumínio, em função de suas espessuras, radiografados simultaneamente com o III metacarpiano. Os autores encontraram coeficientes de correlação, variando de 0,88 a 0,94 , com $\mathrm{P}<0,01$. Utilizaram esta metodologia, também, para acompanhar o crescimento de potros, concluindo que a densitometria óptica, é um método não dispendioso, não invasivo e preciso para estimar o conteúdo mineral ósseo do III metacarpiano de eqüinos em crescimento.

Trabalhando com a fotometria radiográfica VULCANO (2001) desenvolveu e padronizou a técnica da densitometria óptica em imagem radiográfica, que avaliou de forma eficiente, prática e economicamente viável a densidade mineral óssea (DMO), em mílimetros de alumínio (mmAl), do acessório do carpo de eqüinos da raça PSI. Esta técnica proporcionou, com a avaliação do condicionamento físico que vem sendo hoje realizado rotineiramente nos animais de esporte, uma análise mais eficiente do potencial esportivo destes animais. GODOY (2002) utilizou a mesma técnica em cavalos da raça Brasileiro de Hipismo encontrando média de DMO de 4,70 $\pm 0,12 \mathrm{mmAl}$ para os machos e de 4,64 $\pm 0,11 \mathrm{mmAl}$ para as fêmeas.

Levando em consideração as divergências na literatura (O’CALLAGHAN, 1991) sobre a validade da densitometria preventiva, pela variação nos valores entre os diferentes métodos, este estudo objetivou padronizar os valores normais da densidade mineral óssea (DMO) em milímetros de alumínio (mmAl), por meio da densitometria óptica radiográfica, do carpo acessório de eqüinos da raça Quarto de Milha, em treinamento, e sua eficácia na relação custo-benefício, utilizando da mesma técnica e método de VULCANO (2001)e GODOY (2002).

\section{MATERIAL E MÉTODOS}

Foram utilizados 60 eqüinos da raça Quarto de Milha, sendo 30 machos e 30 fêmeas, com idade variando entre 42 e 48 meses, criados na região de Bauru, Estado de São Paulo, clinicamente saudáveis, alimentados com ração comercial, feno de coast-cross ou feno de alfafa, sal mineral e água ad libidum.

Para obtenção das radiografias, utilizou-se aparelho de raios-X portátil modelo FNX Jockey 90a, chassi de tamanho $24 \times 30 \mathrm{~cm}$, contendo filme radiográfico de marca $B R A F^{b}$. O chassi radiográfico era inserido em um porta-chassi confeccionado em madeira compensada com espessura de $3 \mathrm{~mm}$, no qual se fixou uma escala de alumínio (penetrômetro) composta por 29 degraus, com área de $5 \times 15 \mathrm{~mm}$, tendo o primeiro a espessura de $1 \mathrm{~mm}$, variando a seguir de 1 em $1 \mathrm{~mm}$ até o vigésimo nono degrau.

Os fatores de exposição utilizados foram 56 $\mathrm{kVp}$ e $5 \mathrm{mAs}$, com distância foco-filme de $70 \mathrm{~cm}$. A projeção radiográfica utilizada foi a látero-medial, que proporcionou imagem do osso acessório do carpo, juntamente com o penetrômetro, para avaliação da densidade mineral óssea. Os filmes radiográficos foram revelados em processadora automática

A DMO foi determinada, utilizando-se scanner $^{\mathrm{d}}$, com adaptador para transferência HPe ${ }^{\mathrm{e}}$, para digitalização das imagens radiográficas do acessório do carpo. Em seguida, um programa computacional ${ }^{\mathrm{f}}$ foi utilizado para a determinação e análise da densidade óptica das imagens digitalizadas do acessório do carpo e do penetrômetro permitindo a padronização da densidade mineral óssea em milímetros de alumínio (mmAl).

As análises estatísticas dos resultados foram realizadas pelo teste " $\mathrm{t}$ " de Student, para dois grupos independentes (ZAR, 1984).

\section{RESULTADOS E DISCUSSÃO}

Na avaliação da DMO, utilizou-se da técnica de densitometria óptica em imagem radiográfica que se baseia no estudo com uma escala de referência, sendo o alumínio utilizado como matéria-prima padrão para a sua confecção e posterior obtenção de resultados em valores equivalentes em milímetros de alumínio (mmAl), técnica esta similar a utilizada por PLOTNICK et al. 
(1970), MEAKIM et al. (1981), TROUERBACH et al. (1984), MARTIN et al. (1990), SEO et al. (1994), YANG et al. (1994), HAYASHI et al. (1996), VULCANO et al. (2000), VULCANO (2001) e GODOY (2002). A escala foi utilizada como referência para a calibragem do sistema de comparação entre as densidades evitando desta forma as possíveis variáveis que este método poderia apresentar, minimizando, assim, as diferenças no padrão radiográfico entre os filmes analisados que permitiu obter resultados mais precisos (PLOTNICK et al., 1970).

Os valores da DMO em mm Al padronizados por esta técnica na raça Quarto de Milha propiciará a sua utilização na rotina dos exames clínicos dos animais de esporte e trabalho com intuito de analisar a resistência das estruturas ósseas, prevenindo possíveis fraturas durante o seu treinamento ou trabalho. Também permitirá que seja realizada uma avaliação preventiva da DMO nestes animais, sem perder a importância de sua avaliação frente aos valores da DMO pelos diferentes métodos na literatura (O`CALLAGHAN, 1991), uma vez que a metodologia utilizada foi a mesma utilizada por (VULCANO, 2001; GODOY, 2002).

A afirmação de JEFFCOTT (1990), de que o osso trabecular apresentava uma velocidade de modificação oito vezes maior que o cortical, foi considerado importante para este estudo, buscando assim avaliar a estrutura óssea com maior estrutura trabecular e uma cortical delgada, optando-se assim pelo osso acessório do carpo que possui esta característica morfológica (VULCAN0, 2001). Apesar de SCOTTI \& JEFFCOTT (1988), terem utilizado o osso calcâneo (tarso fibular) em estudo in vitro, optou-se pelo primeiro, levando-se em conta a facilidade para a execução das radiografias no membro anterior (GODOY, 2002).

Neste estudo, as fêmeas com média de idade de $43 \pm 2$ meses, apresentaram valores da densidade mineral óssea de 4,49 $\pm 0,69 \mathrm{mmAl}$ (Tabela 1 ), enquanto os machos com idade $45 \pm 2$ meses valores de 4,43 \pm 0,81mmAl (Tabela 2). Também a variável sexo não mostrou diferença significativa entre os valores da DMO, bem como a idade dentro dos grupos, na faixa etária avaliada. Estes resultados são semelhantes aos obtidos por VULCANO (2001), que trabalhou com machos e fêmeas da raça Puro Sangue Inglês, encontrando valores de DMO para as fêmeas de 4,58 \pm
Tabela 1 - Densidade mineral óssea (DMO), em milímetros de alumínio (mmAl), determinada por densitometria óptica em imagem radiográfica, em eqüinos, fêmeas, da raça Quarto de Milha, com idade entre 42 e 48 meses de idade, média e desvio padrão. Bauru, SP., 2003.

\begin{tabular}{|c|c|c|}
\hline Animais & Idade/Meses & $\mathrm{DMO} / \mathrm{mmAl}$ \\
\hline 01 & 42 & 4,77 \\
\hline 02 & 42 & 3,90 \\
\hline 03 & 45 & 4,42 \\
\hline 04 & 43 & 4,29 \\
\hline 05 & 42 & 4,39 \\
\hline 06 & 44 & 4,33 \\
\hline 07 & 42 & 4,57 \\
\hline 08 & 44 & 5,40 \\
\hline 09 & 42 & 4,12 \\
\hline 10 & 48 & 4,41 \\
\hline 11 & 42 & 4,42 \\
\hline 12 & 42 & 2,89 \\
\hline 13 & 44 & 4,70 \\
\hline 14 & 47 & 4,28 \\
\hline 15 & 42 & 4,27 \\
\hline 16 & 43 & 3,89 \\
\hline 17 & 43 & 3,92 \\
\hline 18 & 42 & 4,46 \\
\hline 19 & 48 & 3,79 \\
\hline 20 & 44 & 4,60 \\
\hline 21 & 43 & 4,60 \\
\hline 22 & 48 & 4,75 \\
\hline 23 & 43 & 4,86 \\
\hline 24 & 45 & 5,86 \\
\hline 25 & 46 & 3,97 \\
\hline 26 & 42 & 4,22 \\
\hline 27 & 48 & 4,32 \\
\hline 28 & 43 & 6,83 \\
\hline 29 & 48 & 5,25 \\
\hline 30 & 42 & 4,25 \\
\hline Média & 43 & 4,49 \\
\hline Desvio Padrão & 2 & 0,69 \\
\hline
\end{tabular}

0,40mmAl, com idade de 24,95 $\pm 0,87$ meses e para os machos de 4,46 \pm 0,36mmAl, com idade de 25,13 \pm 0,96 meses, idade em que a epífise distal do rádio apresentava-se fechada e os de GODOY (2002), que avaliou eqüinos da raça Brasileiro de Hipismo onde 12 machos e 12 fêmeas, em crescimento que apresentaram média de DMO respectivamente de 4,70 $\pm 0,12 \mathrm{mmAl} \mathrm{e}$ $4,64 \pm 0,11 \mathrm{mmAl}$.

Ciência Rural, v.36, n.2, mar-abr, 2006. 
Tabela 2 - Densidade mineral óssea (DMO), em milímetros de alumínio (mmAl), determinada por densitometria óptica em imagem radiográfica, em eqüinos, machos, da raça Quarto de Milha, com idade entre 42 e 48 meses de idade, média e desvio padrão. Bauru, SP., 2003.

\begin{tabular}{|c|c|c|}
\hline Animais & Idade/Meses & $\mathrm{DMO} / \mathrm{mmAl}$ \\
\hline 01 & 43 & 4,05 \\
\hline 02 & 44 & 3,77 \\
\hline 03 & 47 & 4,69 \\
\hline 04 & 47 & 4,06 \\
\hline 05 & 42 & 4,91 \\
\hline 06 & 45 & 4,25 \\
\hline 07 & 42 & 3,80 \\
\hline 08 & 48 & 4,54 \\
\hline 09 & 48 & 4,98 \\
\hline 10 & 48 & 4.54 \\
\hline 11 & 48 & 4,98 \\
\hline 12 & 46 & 4,71 \\
\hline 13 & 47 & 4,42 \\
\hline 14 & 47 & 4,30 \\
\hline 15 & 48 & 4,28 \\
\hline 16 & 42 & 4,19 \\
\hline 17 & 45 & 3,53 \\
\hline 18 & 45 & 4,40 \\
\hline 19 & 44 & 2,98 \\
\hline 20 & 42 & 3,54 \\
\hline 21 & 43 & 4,48 \\
\hline 22 & 45 & 4,29 \\
\hline 23 & 42 & 6,32 \\
\hline 24 & 43 & 4,17 \\
\hline 25 & 48 & 5,70 \\
\hline 26 & 45 & 4,48 \\
\hline 27 & 47 & 4,96 \\
\hline 28 & 43 & 4,62 \\
\hline 29 & 44 & 3,86 \\
\hline 30 & 42 & 4,66 \\
\hline Média & 45 & 4,43 \\
\hline Desvio Padrão & 2 & 0,81 \\
\hline
\end{tabular}

\section{CONCLUSÃO}

Este estudo permitiu concluir que a técnica utilizada para avaliar a densidade mineral óssea (DMO) na raça Quarto de Milha, pode ser instituída como rotina na clínica de eqüinos. Em especial, no acompanhamento preventivo da DMO dos animais a campo, em treinamento, que necessitam de uma avaliação mais acurada da sua estrutura óssea decorrente das exigências dos exercícios físicos a que são submetidos, tendo como base os valores normais DMO em mmAl como padrão para a raça Quarto de Milha na faixa etária estudada.

\section{FONTESDEAQUISIÇÃO}

a - FNX Jockey 90 - Distribuidora de Equipamentos Médicos Ltda. - RJ.

b -BRAF - Kodak Brasileira Com. e Ind. Ltda.

c - Macrotec - Ind. e com. de Equipamentos Ltda.

d - Hewlett Packard Scanner Scanjet 6C

e - Hewlett Packard ScanjET

f - SAI - sistema de Inteligência Avançada - SJC - SP.

\section{REFERÊNCIAS}

ANDERSON, J.B. et al. A new technique for the measurements of metacarpal density. British Journal of Radiology, v.39, p.443-450, 1966.

GODOY, C.L.B. Avaliação radiográfica do fechamento epifisário da extremidade distal do rádio e padronização dos valores normais da densidade mineral óssea do acessório do carpo por densitometria óptica em imagem radiográfica em eqüinos da raça Brasileiro de Hipismo (BH). 2002. 64f. Tese (Doutorado em Medicina Veterinária) - Faculdade de Medicina Veterinária e Zootecnia, Universidade Estadual Paulista, Campus de Botucatu.

HAYASHI, T. et al. Evaluation of bone density in neawborn infants by computed X-ray densitometry. Journal of Pediatric Gastroenterology and Nutrition, v.23, n.2, p.130-134, 1996.

JEFFCOTT, L.B. Training effects on bone joints. In: INTERNATIONAL CONFERENCE OF EQUINE SPORTS MEDICINE, 1990, Stockholm. Proceedings... Stockholm: The Association for Equine Sports Medicine, 1990. p.14-17.

MARTIN, R.B. et al. Linear calibration of radiographic mineral density using video-digitizing methods. Calcified Tissue International, v.47, p.82-91, 1990.

MEAKIN, D.W. et al. Estimation of mineral content of the equine third metacarpal by radiographic photometry. Journal of Animal Science, v.53, n.4 p.1019-1026, 1981.

O’CALLAGHAN, M.W. A brief look at new tecnologies and their potential application to equine diagnosis. Veterinary Clinics of North America, v.7, p.467-479, 1991.

PLOTNIK, I.J. et al. Study of in vivo radiographic. Journal of Dental Research, v.49, n.5, p.1034-1041, 1970.

SCOTTI, E.; JEFFCOTT, L.B. The hock as a potential site for non-invasive bone measurement. Equine Veterinary Journal Supplements, v.6, p.93-98, 1988.

SEO, S.G. et al. Assessment of bone density in the distal radius with computer assisted X-ray densitometry (CXD). Bone and Mineral, v.27, p.173-182, 1994. 
SCHNEIDER, R. Radiologic methods of evaluating generalized osteopenia. Orthopedic Clinics of North America, v.14, n.4, p.631-651, 1984.

TROUERBACH, W.T. et al. Microdensitometric analysis of interdental bone estructure; the development of a registration method. Dentomaxillofac Dental, v.13, p.27-31, 1984.

VULCANO, L.C. et al. Determinación de los valores normales de la densidad mineral ósea del carpo accesorio de equinos de la raza Puro Sangre Ingles (PSI) por medio de la densitometría óptica en imagen radiográfica. Imagen Veterinaria, v.3, n.6, p.237- 240, 2000.
VULCANO, L.C. Determinação e padronização dos valores normais da densidade mineral óssea (DMO) do carpo acessório de eqüinos em crescimento da raça Puro Sangue Inglês (PSI) por meio da densitometria óptica em imagem radiográfica. 2001. 62f. Tese (Livre-Docência) - Faculdade de Medicina Veterinária e Zootecnia, Universidade Estadual Paulista, Campus de Botucatu.

YANG, S. et al. Radiographic absorciometry for bone mineral measurement of the phalanges: precision and accuracy study. Radiology, v.192, p.857-859, 1994.

ZAR, J.H. Bioestatistical analysis. New Jersey: PrenticeHall, 1984. 718p. 